\title{
Fate control and well-being in Chinese rural people living with HIV: mediation effect of resilience
}

\author{
Nancy Xiaonan Yua, Jianxin Zhang ${ }^{b}$, Amy Y. M. Chowc, Celia H. Y. Chan ${ }^{c}$ and Cecilia L. W. Chan

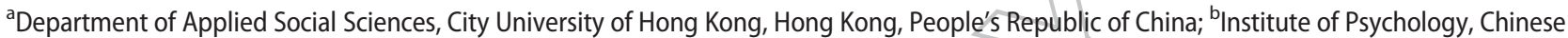 \\ Academy of Sciences, Beijing, People's Republic of China; 'Department of Social Work and Social Administration and Center on Behavioral \\ Health, The University of Hong Kong, Hong Kong, People's Republic of China
}

ABSTRACT

Fate control has been often misconceptualized as a superstitious belief and overlooked in health psychology. It is not known how this cultural belief might impact the well-being of Chinese people living with HIV. This study examined the protective role of fate control for well-being and the potential mediation effect of resilience. Participants in this study were rural patients who contracted HIV via commercial blood donation. In this cross-sectional survey, 250 participants completed measures of fate control, well-being, and resilience. The results showed that fate control and resilience were positively associated with well-being. Resilience mediated the association between fate control and well-being. Our findings provide insight into the adaptive function of fate control as a cognitive defensive mechanism and highlight the need to incorporate this cultural belief in developing culturally sensitive intervention programs for resilience enhancement tailored for this understudied population infected with HIV living in rural China.

\section{Introduction}

Living with HIV is a stressful experience. It is particularly difficult for Chinese villagers who are extremely impoverished and become infected with HIV in the process of donating blood commercially in an effort to ease their financial strain (Wu, Liu, \& Detels, 1995). Influenced by the Buddhist and Taoist philosophies, Chinese people tend to possess a belief in "fate" as an external force to determine life adversities (Leung, 1996). Fate control refers to the belief that a fatalistic force drives life events (Leung, 1996; Leung et al., 2002). This concept consists of two dimensions: fate determinism and fate alterability (Leung et al., 2012). Thus, on top of the predetermined nature of fate, fate is perceived to be alterable using certain means. This belief represents a cognitive defensive mechanism to attribute fate as the cause of misfortune rather than imputing it to one's own fault. This fate-related conceptualization may make people living with HIV (PLHIV) externalize the reason for disease contraction rather than self-blaming, reconstruct their coping strategies, and enhance their well-being. Understanding fate control as a cultural construct among Chinese PLHIV will have significant implications.

Chinese people, particularly those with low literacy living in undeveloped areas, tend to interpret unfortunate life events in terms of fate. Such a fate-related framework helps people to account misfortune to fate, accept misery as predetermined, tolerate sufferings as a necessary part of life, and form positive reappraisal of adversity (Yang, 1996). For example, Chinese cancer patients often viewed fate as one of the causes of cancer and accepted fate as the force to control the disease outcomes (Gonzalez et al., 2015); their fate belief shaped a positive interpretation of the cancer experience (Ching, Martinson, \& Wong, 2009), maintained their mental health (Hou, Lam, \& Fielding, 2009), and facilitated cancer survival (Cheng, Sit, Twinn, Cheng, \& Thorne, 2013). Similarly, fate control was found to be positively correlated with fighting spirit in Chinese cancer patients (Ho, Kam Fung, Chan, Watson, \& Tsui, 2003).

Resilience is defined as the ability to adapt successfully to adversity (Tugade \& Fredrickson, 2004). Its positive functions to prevent the risk for depressive symptoms (Dale et al., 2014) and to promote posttraumatic growth (Murphy \& Hevey, 2013) have been receiving attention in the context of HIV. In Chinese PLHIV, resilience not only was associated with fewer mental health problems (Yu et al., 2009) but also mediated the association between stigma and health status (Zhang et al., 2015). The mediating role of resilience in health psychology

CONTACT Nancy Xiaonan Yu nancy.yu@cityu.edu.hk Department of Applied Social Sciences, City University of Hong Kong, Tat Chee Avenue, Kowloon, HKSAR, People's Republic of China

(C) 2016 Informa UK Limited, trading as Taylor \& Francis Group 
has been documented (Cohen, Baziliansky, \& Beny, 2014; Li, Theng, \& Foo, 2015). Resilience may transmit the positive impacts of protective factors for well-being despite adversities; for example, the presence of protective factors would build resilience, which in turn enhances well-being (Stewart \& Yuen, 2011; Zautra, Hall, \& Murray, \& the Resilience Solutions, 2008). The conceptualization of resilience as ability, rather than process (Jacelon, 1997), informs the development of interventions to enhance resilience as previous studies have shown that resilience is amendable to change (Reivich, Seligman, \& McBride, 2011; Sood, Prasad, Schroeder, \& Varkey, 2011). We developed a psychological intervention tailored for Chinese rural PLHIV that effectively enhanced their resilience, psychosocial well-being, and quality of life (Yu et al., 2014). Although the resilience research has documented affective, cognitive, and behavioral concomitants (Mak, Ng, \& Wong, 2011; Tugade \& Fredrickson, 2004), little has been investigated about spiritual belief (Klasen et al., 2010; Leeson et al,s 2014).

The present study attempts to fill the knowledge gaps by examining fate control and its mechanism of impacting well-being in Chinese rural PLHIV. We hypothesized that (1) fate control and resilience would be positively associated with well-being and (2) resilience would mediate the association between fate control and well-being.

\section{Methods}

\section{Participants}

In rural villages of central China, a substantial number of people were infected with HIV in the process of commercial blood donation (Wu et al., 1995). A total of 250 PLHIV who had a history of commercial blood donation joined this cross-sectional survey. We randomly selected 7 of 22 villages with HIV prevalence greater than 10\% in Henan Province, China. The response rate was 83.3\%. Trained interviewers who were medical staff conducted face-to-face interviews. Participants provided written informed consent before the study began. Ethics approval was obtained from the Ethical Review Board of the City University of Hong Kong. Participants received compensation for their time spent on this survey (CNY30, approximately equivalent to US $\$ 5$ ).

\section{Measures}

Fate control was measured using the eight-item "fate control" subscale of the Social Axioms Scale (Leung et al., 2002; Safdar, Lewis, \& Daneshpour, 2006). This construct consists of two dimensions: fate determinism (e.g., "Fate determines one's success and failure") and
Table 1. Demographic and HIV-related characteristics of participants.

\begin{tabular}{ll}
\hline Sex & $n(\%)$ Mean \pm SD \\
$\quad$ Male & \\
$\quad$ Female & $115(46.0)$ \\
Marital status & $135(54.0)$ \\
$\quad$ Married & $217(86.8)$ \\
$\quad$ Single/divorced/widowed & $33(13.2)$ \\
Age (years) & $46.77 \pm 8.10$ \\
Education (years) & $6.00 \pm 2.24$ \\
Annual family income (RMB) & $2,036.14 \pm 796.40$ \\
Duration since HIV diagnosis (years) & $5.16 \pm 1.26$ \\
\hline Note: 1 US\$ = 6.1RMB. &
\end{tabular}

fate alterability (e.g., "There are certain ways for people to improve their destiny"). Fate control has been used as a single construct (Leung et al., 2002; Safdar et al., 2006) and confirmatory factor analysis in this study found that the single-factor model showed a better fit than the two-factor model. Cronbach's $\alpha$ was .84 in the present study.

Well-being was measured using the three-item Integrative Body-Mind-Spirit Well-being Measurement (e.g., "My moods are positive"), based on the concept of holistic well-being (Lee, Ng, Leung, \& Chan, 2009). This measure is the brief version of the Holistic Wellbeing Scale (Chan et al., 2014). Participants rated their well-being in the past month. Exploratory factor analysis showed that these three items loaded on a single dimension, explaining $66.55 \%$ of the variance. Cronbach's $\alpha$ was .84 in the present study.

Resilience was measured using the 25-item ConnorDavidson Resilience Scale (2003). Participants rated their responses (e.g., "I am able to adapt to change") based on the previous month. The Chinese version (Yu \& Zhang, 2007) had previously been used in a study among Chinese rural PLHIV (Yu et al., 2009). Cronbach's $\alpha$ was .94 in the present study.

\section{Results}

Table 1 shows demographic and HIV-related characteristics of the 250 participants. Most of them were middleaged with low levels of education and family income.

Pearson correlations were computed to estimate the bivariate associations among the study variables. As shown in Table 2, fate control was associated with resilience $(r=.29, p<.01)$ and well-being $(r=.23$, $p<.01)$. Resilience was associated with well-being $(r=.55, p<.01)$.

We applied path analysis using Mplus 7 to test the indirect effect of resilience in the association between fate control and well-being. We also performed a Sobel test using a bootstrap method with 5000 bootstrap 
Table 2. Pearson correlations of study variables.

\begin{tabular}{lcll}
\hline & Mean \pm SD & Fate control & Resilience \\
\hline Fate control & $3.06 \pm .87$ & - & \\
Resilience & $69.19 \pm 17.63$ & $.29^{* *}$ & - \\
Well-being & $5.38 \pm 2.23$ & $.23^{* *}$ & $.55^{* *}$ \\
\hline$* * p<.01$. & & &
\end{tabular}

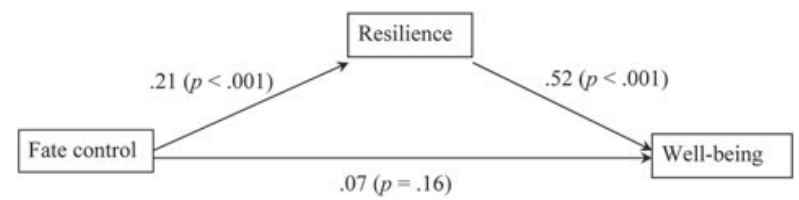

Figure 1. The mediation effect of resilience in the association between fate control and well-being. resamples. The mediation model fit the data well: $\chi^{2}(1)=$ $1.95, p=.16 ; \mathrm{RMSEA}=.06 ; \mathrm{CFI}=.99 ; \mathrm{TLI}=.97 ; \mathrm{SRMR}$ $=.05(\mathrm{Hu} \&$ Bentler, 1999). The total effect of this mediation model was significant $(p<.001)$. The indirect effect of fate control on well-being was significant $(p$ $<.01)$, whereas the direct effect of fate control was not significant $(p>.05)$ when resilience was controlled, indicating the full mediation effect of resilience (Baron \& Kenny, 1986). The bootstrap method generated identical results. The results are shown in Figure 1.

\section{Discussion}

This study presents novel findings about the protective role of fate control for well-being among Chinese PLHIV and explains how resilience works as a mediation mechanism. Fate control used to be misconceptualized as superstitious beliefs, and its adaptive function in health psychology has been overlooked. However, in the context of searching for an explanation for HIV infection, fate control may serve as a cognitive defensive mechanism to reduce self-blaming of PLHIV by attributing to fate which is externally controlled. On the other hand, its alterable nature offers them hope that some actions might alter their fate. In the studied PLHIV whose internal control might not effectively change the disease outcomes, fate control may have helped them to interpret the rationale for HIV infection, make sense of their suffering, and relinquish control over prognosis outcomes to destiny. Hence, fate control serves as a cognitive defensive system to preserve psychological integrity and maintain the sense of coherence of these PLHIV. Our results indicate that it is promising to integrate fate control in developing culturally sensitive resilience enhancement intervention programs for this underserved population. Spiritual therapy targeting fate beliefs has been shown to be effective in improving the quality of life of cancer patients (Jafari et al., 2012).
Several limitations should be noted when interpreting the results. First, using a convenience sample may limit generalization of our results on fate control to those with a higher socioeconomic status. Second, the results should be interpreted with caution considering the weak-to-moderate correlations among the study variables. Third, the associations reported in this cross-sectional study are correlational in nature.

\section{Conclusions}

By identifying resilience as a mechanism to account for protective effects of fate control, the current study provides insight into the adaptive function of this cultural belief in an understudied sample. The findings highlight the need to reexamine fate control in illness management and the potential to incorporate it as a cognitive belief framework in resilience interventions in HIV care.

\section{Disclosure statement}

No potential conflict of interest was reported by the authors.

\section{Funding information}

The work described in this paper was partially supported by a grant from the Research Grants Council of the Hong Kong Special Administrative Region, China [Project No. CityU 21402714]. The sponsor had no further role in study design, in the collection, analysis and interpretation of data, in the writing of the report, and in the decision to submit the paper for publication.

\section{References}

Baron, R. M., \& Kenny, D. A. (1986). The moderator-mediator variable distinction in social psychological research: Conceptual, strategic, and statistical considerations. Journal of Personality and Social Psychology, 51, 11731182. doi:10.1037/0022-3514.51.6.1173

Chan, C. H. Y., Chan, T. H. Y., Leung, P. P. Y., Brenner, M. J., Wong, V. P. Y., Leung, E. K. T., ... Chan, C. L. W. (2014). Rethinking well-being in terms of affliction and equanimity: Development of a holistic well-being scale. Journal of Ethnic \& Cultural Diversity in Social Work, 23, 289-308. doi:10. 1080/15313204.2014.932550

Cheng, H., Sit, J. W., Twinn, S. F., Cheng, K. K., \& Thorne, S. (2013). Coping with breast cancer survivorship in Chinese women: The role of fatalism or fatalistic voluntarism. Cancer Nursing, 36, 236-244. doi:10.1097/NCC. 0b013e31826542b2

Ching, S. S., Martinson, I. M., \& Wong, T. K. (2009). Reframing: Psychological adjustment of Chinese women at the beginning of the breast cancer experience. Qualitative Health Research, 19, 339-351. doi:10.1177/ 1049732309331867 
Cohen, M., Baziliansky, S., \& Beny, A. (2014). The association of resilience and age in individuals with colorectal cancer: An exploratory cross-sectional study. Journal of Geriatric Oncology, 5, 33-39. doi:10.1016/j.jgo.2013.07.009

Connor, K. M., \& Davidson, J. R. (2003). Development of a new resilience scale: The Connor-Davidson Resilience Scale (CD-RISC). Depression and Anxiety, 18, 76-82. doi:10.1002/da.10113

Dale, S. K., Weber, K. M., Cohen, M. H., Kelso, G. A., Cruise, R. C., \& Brody, L. R. (2014). Resilience moderates the association between childhood sexual abuse and depressive symptoms among women with and at-risk for HIV. AIDS and Behavior, 19, 1379-1387. doi:10.1007/s10461-014-0855-3

Gonzalez, P., Lim, J. W., Wang-Letzkus, M., Flores, K. F., Allen, K. M., Castaneda, S. F., \& Talavera, G. A. (2015). Breast cancer cause beliefs: Chinese, Korean, and Mexican American breast cancer survivors. Western Journal of Nursing Research, 37, 1081-1099. doi:10.1177/0193945914541518

Ho, S. M., Kam Fung, W., Chan, C. L., Watson, M., \& Tsui, Y. K. (2003). Psychometric properties of the Chinese version of the Mini-Mental Adjustment to Cancer (MINI-MAC) scale. Psycho-Oncology, 12, 547-556. doi:10.1002/pon.672

Hou, W. K., Lam, W. W., \& Fielding, R. (2009). Adaptation process and psychosocial resources of Chinese colorectal cancer patients undergoing adjuvant treatment: A qualitative analysis. Psycho-Oncology, 18, 936-944. doi:10.1002/pon.1457

Hu, L. T., \& Bentler, P. M. (1999). Cutoff criteria for fit indexes in covariance structure analysis: Conventional criteria versus new alternatives. Structural Equation Modeling, 6, 155. doi:10.1080/10705519909540118

Jacelon, C. S. (1997). The trait and process of resilience. Journal of Advanced Nursing, 25, 123-129. doi:10.1046/j. 1365-2648.1997.1997025123.X

Jafari, N., Zamani, A., Farajzadegan, Z., Bahrami, F., Emami, H., \& Loghmani,A. (2012). The effect of spiritual therapy for improving the quality of life of women with breast cancer: A randomized controlled trial. Psychology, Health \& Medicine, 18, 56-69. doi:10.1080/13548506.2012.679738

Klasen, F., Oettingen, G., Daniels, J., Post, M., Hoyer, C., \& Adam, H. (2010). Posttraumatic resilience in former Ugandan child soldiers. Child Development, 81, 10961113. doi:10.1111/j.1467-8624.2010.01456.x

Lee, M. Y., Ng, S.-M., Leung, P. P. Y., \& Chan, C. L. W. (2009). Integrative body-mind-spirit social work: An empirically based approach to assessment and treatment. New York, NY: Oxford University Press.

Leeson, L. A., Nelson, A. M., Rathouz, P. J., Juckett, M. B., Coe, C. L., Caes, E. W., \& Costanzo, E. S. (2014). Spirituality and the recovery of quality of life following hematopoietic stem cell transplantation. Health Psychology, 34, 920-928. doi:10. 1037/hea0000196

Leung, K. (1996). The role of beliefs in Chinese culture. In M. $\mathrm{H}$. Bond (Ed.), The handbook of Chinese psychology (pp. 246-262). Hong Kong: Oxford University Press.

Leung, K., Bond, M. H., de Carrasquel, S. R., Muñoz, C., Hernández, M., Murakami, F., ... Singelis, T. M. (2002). Social axioms: The search for universal dimensions of general beliefs about how the world functions. Journal of Cross-Cultural Psychology, 33, 286-302. doi:10.1177/0022022102033003005

Leung, K., Lam, B. C. P., Bond, M. H., Conway, L. G., Gornick, L. J., Amponsah, B., ... Zhou, F. (2012). Developing and evaluating the social axioms survey in eleven countries: Its relationship with the five-factor model of personality. Journal of Cross-Cultural Psychology, 43, 833-857. doi:10. 1177/0022022111416361

Li, J., Theng, Y.-L., \& Foo, S. (2015). Does psychological resilience mediate the impact of social support on geriatric depression? An exploratory study among Chinese older adults in Singapore. Asian Journal of Psychiatry, 14, 2227. doi:10.1016/j.ajp.2015.01.011

Mak, W. W., Ng, I. S., \& Wong, C. C. (2011). Resilience: Enhancing well-being through the positive cognitive triad. Journal of Counseling Psychology, 58, 610-617. doi:10.1037/a0025195

Murphy, P., \& Hevey, D. (2013). The relationship between internalised HIV-related stigma and posttraumatic growth. AIDS and Behavior, 17, 1809-1818. doi:10.1007/s10461-013-0482-4

Reivich, K. J., Seligman, M. E. P., \& McBride, S. (2011). Master resilience training in the U.S. Army. American Psychologist, 66, 25-34. doi:10.1037/a0021897

Safdar, S., Lewis, J. R., \& Daneshpour, M. (2006). Social axioms in Iran and Canada: Intercultural contact, coping and adjustment. Asian Journal of Social Psychology, 9, 123131. doi:10.1111/j.1467-839X.2006.00189.x

Sood, A., Prasad, K., Schroeder, D., \& Varkey, P. (2011). Stress management and resilience training among department of medicine faculty: A pilot randomized clinical trial. Journal of General Internal Medicine, 26, 858-861. doi:10.1007/ s11606-011-1640-x

Stewart, D. E., \& Yuen, T. (2011). A systematic review of resilience in the physically ill. Psychosomatics, 52, 199-209. doi:10.1016/j.psym.2011.01.036

Tugade, M. M., \& Fredrickson, B. L. (2004). Resilient individuals use positive emotions to bounce back from negative emotional experiences. Journal of Personality and Social Psychology, 86, 320-333. doi:10.1037/0022-3514.86. 2.320

Wu, Z., Liu, Z., \& Detels, R. (1995). HIV-1 infection in commercial plasma donors in China. Lancet, 346, 61-62. doi:10.1016/S0140-6736(95)92698-4

Yang, K. S. (1996). Theories and research in Chinese personality: An indigenous approach (Vol. 19). New Delhi: Sage.

Yu, X., Lau, J. T. F., Mak, W. W. S., Cheng, Y., Lv, Y., \& Zhang, J. (2014). A pilot theory-based intervention to improve resilience, psycho-social well-being and quality of life among people living with HIV in rural China. Journal of Sex \& Marital Therapy, 40, 1-16. doi:10.1080/0092623X.2012.668512

Yu, X., Lau, J. T. F., Mak, W. W. S., Cheng, Y. M., Lv, Y. H., \& Zhang, J. X. (2009). Risk and protective factors in association with mental health problems among people living with HIV who were former plasma/blood donors in rural China. AIDS Care, 21, 645-654. doi:10.1080/09540120802459770

Yu, X., \& Zhang, J. (2007). Factor analysis and psychometric evaluation of the Connor-Davidson Resilience Scale (CDRISC) in Chinese people. Social Behavior and Personality, 35, 19-30. doi:10.2224/sbp.2007.35.1.19

Zautra, A. J., Hall, J. S., Murray, K. E., \& the Resilience Solutions, G. (2008). Resilience: A new integrative approach to health and mental health research. Health Psychology Review, 2, 41-64. doi:10.1080/17437190802298568

Zhang, L., Li, X., Qiao, S., Zhou, Y., Shen, Z., Tang, Z., ... Stanton, B. (2015). The mediating role of individual resilience resources in stigma-health relationship among people living with HIV in Guangxi, China. AIDS Care, 27, 13171325. doi:10.1080/09540121.2015.1054338 